\title{
Urheberrechtsschutz für Software
}

\author{
BGH, Urteil.vom 14. Juli 1993 (I ZR 47/91) - "Buchbaltungsprogramm"
}

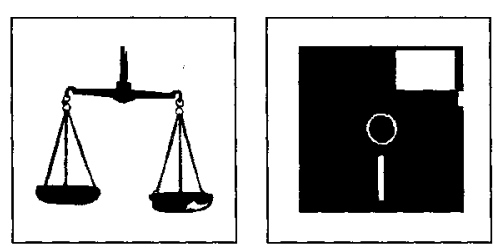

\section{Leitsätze}

a) Zur Frage der Urheberrechtsschutzfähigkeit eines Buchhaltungsprogramms.

b) Die Angabe der Initialen eines Namens in der Kopfleiste der Bildschirmmaske eines Computerprogramms reicht in der Regel aus, um die Urheberschaftsvermutung nach $\S 10$ UrhG zu begründen.

c) Die Miturheberschaft nach $\S 8$ UrhG setzt auch bei einem Computerprogramm ein gemeinsames Schaffen der Beteiligten voraus, bei dem jeder einen schöpferischen Beitrag zum einheitlichen Schöpfungsproze $B$ der Werkvollendung leistet.

\section{Tatbestand}

Der Kläger ist Inhaber einer Firma, die sich mit der Entwicklung und Verbreitung von Computerprogrammen befaßt. Die Beklagte berät Unternehmen in Fragen der Organisation und Datenverarbeitung. Die Parteien streiten über die Urheberschaft an einem Computerprogramm.

Der Kläger begann im Jahre 1975 mit der Entwicklung des Finanzbuchhaltungsprogramms RT-FIBU. Von November 1980 bis 1984 arbeiteten die Parteien mit dem Ziel zusammen, das Programm zu vermarkten. Es wurde in dieser Zeit weiterentwickelt, wobei Streit darüber besteht, wer den maßgeblichen Anteil erbracht hat. Die Parteien installierten RT-FIBU-Programme bei verschiedenen Firmen, ohne daß es zwischen ihnen zu einer vertraglichen R'egelung gekommen war.

Nach Beendigung der Zusammenarbeit verkaufte die Beklagte im Jahre 1985 ein RT-FIBUProgramm an eine Lörracher Firma.

Der Kläger hat darin (u. a.) eine Urheberrechtsverletzung gesehen und die Beklagte auf Unterlassung, Auskunftserteilung und Feststellung einer Schadensersatzpflicht in Anspruch genommen. Eine von der Beklagten erhobene Widerklage ist in den Vorinstanzen rechtskräftig abgewiesen worden.

Der Kläger hat die Ansicht vertreten, das Programm RT-FIBU sei urheberrechtlich geschützt. Er sei auch allein Urheber dieses Programms. Die Programmbearbeitung zur Anpassung an die Bedürfnisse der Kunden der Beklagten sei nur durch ihn erfolgt. Die Be-

- klagte habe lediglich die Kundenwünsche in Form sogenannter Generierungsanweisungen an ihn weitergegeben, die er im Programm verwirklicht habe.

Die Beklagte ist dem entgegengetreten. Sie hat vorgebracht, das RT-FIBU-Programm sei nicht schutzfähig, da es aus vorbekannten Elementen bestehe. Im übrigen sei das Programm erst unter ihrer maßgeblichen Mitarbeit zur Lauffähigkeit entwickelt worden. Die R'olle des Klägers habe sich im wesentlichen darauf beschränkt, nach den detaillierten Vorgaben ihres Mitarbeiters $H$. zu programmieren. Das bei der Lörracher Firma installierte Programm sei nicht mit dem des Klägers identisch.

Das Landgericht hat die Klage nach Beweisaufnahme abgewiesen. Die Berufung des Klägers ist ohne Erfolg ge lieben. Mit der R'evision verfolgt der Kläger seine Klageansprüche weiter. Die Beklagte beantragt, die R'evision zurückzuweisen.

\section{Entscheidungsgründe}

I. Das Berufungsgericht hat urheberrechtliche Ansprüche gemäß $\$ 97$ UrhG verneint und dazu ausgeführt: Das in der Zeit von 1977 bis 1984 entstandene RT-FIBU-Programm genieße zwarnnach $\$ 2$ Abs. 1 Nr. 1 UrhG Urheberrechtsschutz; denn es stelle nach den Ausführungen des gerichtlichen Sachverständigen hinsichtlich der Gestaltung der Fremdwährungsbuchhaltung, der Datenstrukturen und der Bildschirmgestaltung eine eigenschöpferische Leistung dar, die sich vom durchschnittlichen Schaffen hinreichend abhebe. Der Kläger sei jedoch nicht als Schöpfer des Programms anzusehen. Denn er habe die schutzbegründenden Programmelemente lediglich auf Anweisung des Mitarbeiters H. der Beklag- ten programmiert. Insoweit werde auf die Beweiswürdigung des Landgerichts Bezug genommen, das die Aussage des als Zeugen vernommenen Mitarbeiters eingehend und zutreffend gewürdigt habe.

Die Parteien

Die Entwicklung von RT-FIBU

Das Begebren des Klägers

Die Position des Klägers

Die Position der Beklagten

Prozeßgeschichte

OLG Hamburg:

Kläger nicht Schöpfer des

Programms 


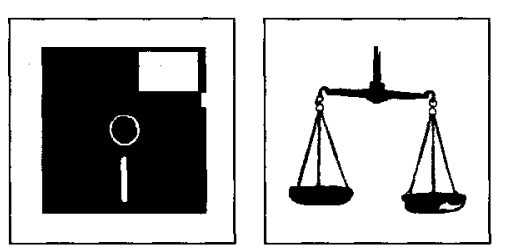

Revision erfolgreich

Das streitgegenständliche Programm

Urbeberrechtsschutzfühigkeit des Programms

Nene Lage nach der Anderung des Urbeberrechts

Der Kläger habe im übrigen nicht substantiiert vorgetragen, daß bereits eine vor Beginn der Zusammenarbeit der Parteien von ihm geschaffene Programmversion urheberrechtsschutzfähig gewesen sei. Es könne deshalb auch nicht davon ausgegangen werden, daß eine vom Kläger vorher erbrachte schöpferische Leistung Eingang in das spätere Programm gefunden habe.

II. Die gegen diese Beurteilung gerichteten Angriffe der Revision haben Erfolg. Sie führen zur Aufhebung und Zurückverweisung.

Die Verneinung einer von der Beklagten begangenen Urheberrechtsverletzung durch das Berufungsgericht hält der revisionsrechtlichen Nachprüfung nicht stand.

1. Streitgegenstand ist das RT-FIBU-Programm in der Version 2.02 (vgl. Klageschrift S. 10 und Ausdruck der Maske gemäß Anlage K 6 sowie Benutzerhandbuch gemäß Anlage K 10), dessen Verletzung der Kläger behauptet, indem er vorträgt, die Beklagte habe dieses Programm nach Beendigung der Zusammenarbeit zwischen den Parteien bei einer Lörracher Firma installiert (vgl. Klageschrift S. 10 und Ausdruck der Maske gemäß Anlage K 7). Nur dieses Programm ist daraufhin zu überprüfen, ob es urheberrechtsschutzfähig ist, ob der Kläger (Mit-)Urheber ist und ob das von der Beklagten vertriebene Programm damit übereinstimmt. Dagegen kommt es für die Prüfung in der Revisionsinstanz nicht darauf an, ob auch frühere Programmversionen des Klägers aus der Zeit vor der Zusammenarbeit der Parteien urheberrechtsschutzfähig sind. Insoweit ist das Berufungsgericht (BU 13 unten) von der Revision unbeanstandet davon ausgegangen, daß es an einem substantiierten Vorbringen des Klägers und auch an sonstigen Anhaltspunkten für eine Urheberrechtsschutzfähigkeit fehlt. Deshalb ist auch die Frage, ob sich das streitgegenständliche Programm als eine Vervielfältigung oder Bearbeitung früherer schutzfähiger Programmversionen des Klägers darstellt, einer revisionsrechtlichen Nachprüfung nicht zugänglich.

2. Ohne Rechtsverstoß und von der Revision - als für sie günstig - unbeanstandet hat das Berufungsgericht dem streitgegenständlichen RT-FIBU-Computerprogramm gemäß $\$ 2$ Abs. 1 Nr. 1 UrhG Urheberrechtsschutz zugebilligt.

a) Das Berufungsgericht ist dabei von den rechtlichen Anforderungen ausgegangen, die der Senat bislang zur Urheberrechtsschutzfähigkeit von Computerprogrammen aufgestellt hat (vgl. BGHZ 94, 276, 281 ff. - Inkassoprogramm¹; 112, 264, 273 f. - Betriebssystem²); d. h., es hat eine deutlich über dem durchschnittlichen Programmiererschaffen liegende eigene schöpferische Leistung verlangt, die ihren Niederschlag und Ausdruck in der Auswahl, Sammlung, Anordnung und Einteilung der Informationen finden muß.

Nach der durch das Zweite Gesetz zur Änderung des Urheberrechtsgesetzes vom 9. Juni 1993 (BGBl. I 910) ${ }^{3}$ erfolgten Umsetzung der EG-Richtlinie vom 14. Mai 1991 über den Rechtsschutz von Computerprogrammen (ABl. EG Nr. L 122 S. 42) ${ }^{4}$ werden allerdings künftig bei Computerprogrammen geringere Schutzanforderungen $\mathrm{zu}$ stellen sein (vgl. \69 a Abs. 3 UrhG n. F. = Art. 1 Abs. 3 der Richtlinie ${ }^{5}$; dazu Erdmann/Bornkamm GRUR 1991, 877 ff.; Dreier CR 1991, 577, 578; Lebmann GRUR Int. 1991, 327, 328 f. und NJW 1991, 2112, 2113; Ullmann CR 1992, 641, 642 f. ). Dies gilt nach $\$ 137$ d Abs. 1 Satz 1 UrhG n. F. ${ }^{6}$ auch für die vor dem Inkrafttreten der Gesetzesänderung (24. Juni 1993) geschaffenen Computerprogramme. Die Rückwirkung erstreckt sich jedoch nicht auf die vor diesem Zeitpunkt liegenden Verletzungshandlungen, die grundsätzlich - was vor ạllem für die Schadensersatzansprüche, nicht dagegen auch für die in die Zukunft gerichteten Unterlassungsansprüche von Bedeutung ist - nach altem Recht $\mathrm{zu}$ beurteilen sind. $\mathrm{Ob}$ es angesichts der Änderung der Rechtslage zulässig ist, die strengeren Anforderungen der bisherigen Rechtsprechung auch bei der Beurteilung von Altfällen zu lockern und die Auslegung näher an die erfolgte Rechtsvereinheitlichung heranzuführen, kann vorliegend auf sich beruhen, weil das Berufungsgericht hier bereits auf der Grundlage der vom Senat bislang aufgestellten Anforderungen ohne Rechtsverstoß zur Urheberrechtsschutzfähigkeit des streitgegenständlichen Computerprogramms gelangt ist.

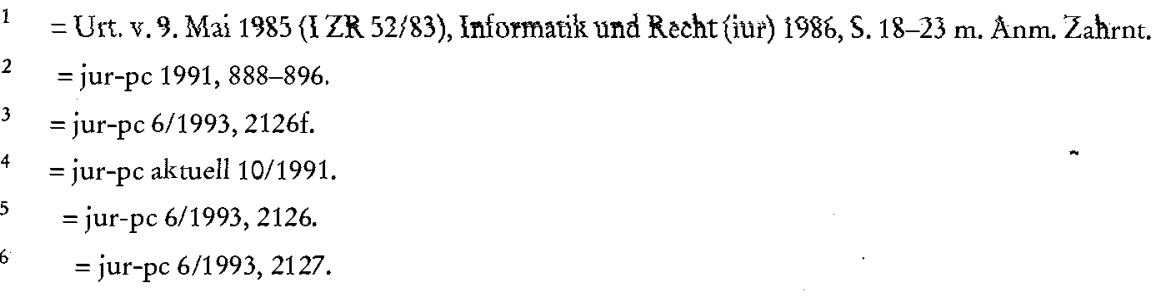


b) Das Berufungsgericht hat - gestützt auf die mündlichen und schriftlichen Ausführungen des gerichtlichen Sachverständigen - festgestellt, daß das RT-FIBU-Computerprogramm hinsichtlich der Gestaltung der Fremdwährungsbuchhaltung, der Datenstrukturen und der Bildschirmgestaltung insgesamt eine eigenschöpferische Leistung darstellt, die sich von dem Durchschnittsschaffen hinreichend abhebe. Demgegenüber rügt die Revision - was für die Frage der Miturheberschaft von Bedeutung ist - mit Erfolg, daß diese Kriterien nicht vollständig sind, weil der Sachverständige in seinem Gutachten (S. 27) nicht nur die Datenstrukturen als individuell und qualitativ hochstehend bezeichnet hat, sondern auch die Struktur und die Aufteilung des Programms selbst. Im übrigen werden die tatrichterlichen Feststellungen von den Parteien aber nicht angegriffen und sind daher der Prüfung in der Revisionsinstanz zugrunde zu legen.

Die weitere Annahme des Berufungsgerichts, daß der Sachverständige nicht - wie vom Kläger anfangs behauptet - eine Programmversion aus dem Jahre 1977, sondern ein in der Zeit von 1974 bis 1984 entstandenes Programm untersucht hat, beruht vor allem auf den Ausführungen auf S. 11 des Gutachtens und wird in der Revisionsinstanz nicht mehr in Zweifel gezogen.

3. Die gegen die Verneinung jeglicher Urheberschaft des Klägers an dem streitgegenständlichen Computerprogramm gerichteten Angriffe der Revision haben Erfolg. Der Kläger ist gemeinsam mit dem Zeugen $\mathrm{H}$. als Miturheber anzusehen.

a) Die Annahme einer Miturheberschaft setzt in rechtlicher Hinsicht ein gemeinsames Schaffen der Beteiligten voraus, bei dem jeder einen schöpferischen Beitrag leistet, der in das gemeinsame Werk einfließt (vgl. BGH, Urt. v. 19. 10. 1962 - I ZR 174/60, GRUR 1963, 40, 41 - Straßen - gestern und morgen; BGH, Urt. v. 6.2. 1985 - 1 ZR 179/82, GRUR 1985, 529 - Happening). Die schöpferische Mitwirkung kann bei einem stufenweise entstehenden Werk - wie z. B. einem Computerprogramm - auch in einem Vorstadium erfolgen, wenn sie als unselbständiger Beitrag zum einheitlichen Schöpfungsprozeß der Werkvollendung geleistet wird (vgl. BGHZ 94, 276, 281 f., 284, 288 - Inkassoprogramm) ${ }^{7}$. Die einzelnen Miturheber brauchen nicht jeden Beitrag zum gemeinsamen Werk auch gemeinsam zu erbringen; es reicht aus, daß jeder in Unterordnung unter die gemeinsame Gesamtidee einzelne (schöpferische) Beiträge selbst erbringt (vgl. BGH, Urt. v. 3.3.1959 - I ZR 17/58, GRUR 1959, 335, 336 - Wenn wir alle Engel wären).

b) Die tatrichterliche Feststellung des Berufungsgerichts, der Kläger habe keinen solchen Beitrag zur Entstehung des streitgegenständlichen Computerprogramms geleistet, wird von der Revision zu Recht als verfahrensfehlerhaft ( $\$ 286 \mathrm{ZPO})$ gerügt.

Das Berufungsgericht hat nicht geprüft, ob der Kläger die Urheberschaftsvermutung des $\$ 10$ Abs. 1 UrhG für sich in Anspruch nehmen kann. Das ist an sich nicht zu beanstanden, da das Berufungsgericht positiv festgestellt hat, daß der Kläger nicht Urheber ist. Mit Erfolg macht aber die Revision geltend, daß das Berufungsgericht diese Feststellung nicht rechtsfehlerfrei getroffen hat.

Nach $₫ 10$ Abs. 1 UrhG wird derjenige, der auf den Vervielfältigungsstücken eines erschienenen Werkes in der üblichen Weise als Urheber bezeichnet ist, bis zum Beweis des Gegenteils auch als Urheber angesehen. Die in der üblichen Weise erfolgte Urheberbezeichnung begründet eine tatsächliche Vermutung dafür, daß der solchermaßen Bezeichnete der Urheber ist, d. h. der Schöpfer des Werkes (BGH, Urt. v. 7.6.1990 - I ZR 191/88, GRUR 1991, 456, 457 - Goggolore). Diese Vermutung kann der Kläger hier für sich in Anspruch nehmen. Er ist u. a. in der Kopfleiste der Maskenausdrucke gemäß Anlage K 6 und 7 (dort jedenfalls auf den ersten beiden Blättern) mit den Initialen A.S.W. (= A. S. W. Wnd $_{1}$ in der Fußzeile des Bedienungshandbuchs gemäß Anlage K 10 mit der Angabe "Copyright A.S.W." als Urheber ausgewiesen. Der Senat vermag aus eigener Kenntnis zu beurteilen, daß diese Benennung in einer bei Computerprogrammen üblichen Weise erfolgt ist.

Diese für die Urheberschaft des Klägers sprechende Vermutung kann aufgrund der vom Berufungsgericht getroffenen und von ihm in Bezug genommenen Feststellungen des Landgerichts nicht als widerlegt angesehen werden. Das Berufungsgericht hat zum einen dabei nicht hinreichend beachtet, daß es bei einem Streit über die (Mit-)Urheberschaft bei mehreren Beteiligten mit unterschiedlichen Arbeitsleistungen einer näheren Prüfung und Differenzierung der schöpferischen Elemente bedarf (vgl. BGHZ 94, 276, 284 - Inkasso-

Urbeberschaftsvermutung des $\$ 10$ Abs. 1 UrbG

Die Namensangabe auf den Vervielfältigungsstücken

\footnotetext{
= Urt. v. 9. Mai 1985 (I ZR 52/83), Informatik und Recht (iur) 1986, S. 18-23, 19f., 21, 22.
} 


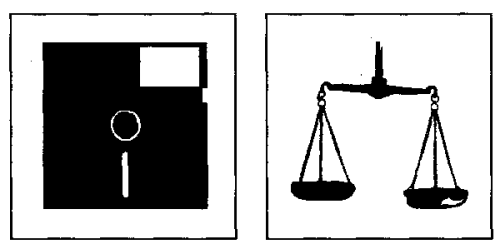

Nicht relevant: Umfang und Größe der Beiträge

Der Programmteil "Fremdwäbrungsbucbung"

Individuelle Züge der Datenstrukturierung

Überdurchschnittliche Programmstruktur programm). ${ }^{8}$ Sodann hat es - was die Revision rügt - bei seiner Beweiswürdigung den Sach- und Streitstand nicht voll ausgeschöpft. Dies kann das Revisionsgericht jedoch nachholen, ohne daß es insoweit einer Aufhebung und Zurückverweisung bedarf.

Es ist nicht erforderlich, daß der Kläger an allen schöpferischen Elementen mitgewirkt hat. Es kommt deshalb auf den Umfang und die Größe der Beiträge nicht an, sofern sie nur schöpferischer Art sind. Dies läßt sich jedenfalls hinsichtlich der Gestaltung der Fremdwährungsbuchhaltung, der Datenstrukturen und der Programmstruktur nicht ausschließen. Hinsichtlich der Fremdwährungsbuchung ist zu berücksichtigen, daß sie schon in der ursprünglichen Programmversion des Klägers enthalten war, so daß es maßgebend darauf ankommt, ob der Zeuge H. sie völlig neu gestaltet und den Kläger dabei lediglich als unselbständiges Werkzeug eingesetzt hat oder ob es sich um eine Bearbeitung im schöpferischen Bereich handelt. Insoweit ist weder dem Berufungsurteil noch dem landgerichtlichen Urteil, auf dessen Beweiswürdigung das Berufungsgericht gemäß $\$ 543$ Abs. 1 ZPO in zulässiger Weise Bezug genommen hat, etwas zu entnehmen. Der gerichtliche Sachverständige hat die - auch anders lösbare - konkrete Ausgestaltung der Fremdwährungsbuchung in der Form des direkten Auftauchens auf dem Bildschirm (ohne Aufruf eines Untermenüs) als originell und eigenschöpferisch angesehen; die Art der Einbeziehung sei gut und überraschend und stelle in der gewählten Realisierungsform eine überdurchschnittliche Leistung dar (Gutachten S. 24 und 25 = GA 1158 f. sowie Vernehmungsprotokoll GA I 203, 207 f.). Aus der Vernehmung des Zeugen $H$. ergibt sich nicht hinreichend, daß diese eigenschöpferischen Züge ausschließlich auf seine Mitwirkung zurückzuführen sind. Die Vorinstanzen haben dazu lediglich festgestellt, der Zeuge habe bekundet, die Fremdwährungsbuchung umgestaltet und dem Kläger die einzelnen Schritte - Dateierweiterung, Plausibilitätsprüfung und Auswertungsmöglichkeit - vorgegeben zu haben; der Kläger habe diese Schritte dann in die Programmiersprache umgesetzt (LGU 14 f.). Die von dem Zeugen in diesem Zusammenhang beispielhaft angeführte Einbeziehung der deutschen Umsatzsteuer-Voranmeldung, die vom Kläger praktisch nur noch auf dem Bildschirm habe "abgetippt" zu werden brauchen (LGU 14, Vernehmungsprotokoll GA II 262), macht deutlich, daß diese Vorgaben eher dem wissenschaftlich-technischen Gedankengut zuzuordnen sind, das frei und jedermann zugänglich bleiben muß und daher dem Urheberrechtsschutz grundsätzlich nicht unterfällt (vgl. BGHZ 94, 276, 285 - Inkassoprogramm) ${ }^{9}$ Der Zeuge hat überdies die Frage, ob er an der Umsetzung des Programms über die Zielvorgaben hinaus mitgewirkt habe, verneint und erklärt, er habe keine Statements geändert und auch nicht programmiert. Danach kann nicht davon ausgegangen werden, daß allein die Mitwirkung des Zeugen und nicht auch die des Klägers in den vom Sachverständigen als eigenschöpferisch gekennzeichneten Bereich, nämlich der Art und Weise der Einbeziehung der Fremdwährungsbuchhaltung in das Programm, fällt.

Der Datenstrukturierung hat der Sachverständige jedenfalls in der Kombination mit den anderen schöpferischen Elementen noch individuelle Züge beigemessen (Gutachten S. 15 und $27=$ GA I 149 und 161 sowie Vernehmungsprotokoll GA I 206). Er hat die Strukturierung der näher bezeichneten Daten durch verkettete Listen und Indices mit Zeigern zwar als nicht neu und zum Standardwerkzeug beim Umgang mit größeren Datenmengen bezeichnet. Neu sei zum damaligen Zeitpunkt wohl nur die Verwendung dieser Datenstruktur innerhalb eines Programms zur Finanzbuchhaltung gewesen. Wenn die Vörinstanzen - den Ausführungen des Sachverständigen folgend - die Datenstruktur als schutzbegründend mitherangezogen haben, so hätten sie auch berücksichtigen müssen, daß der Zeuge insoweit in erster Linie nur die Änderung der Belegnummerndatei für sich in Anspruch genommen hat (LGU 14, Vernehmungsprotokoll GA II 267). Darin erschöpft sich die Datenstrukturierung aber nicht.

Schließlich hat der Sachverständige - von den Vorinstanzen unberücksichtigt - auch die Programmstruktur mit der Aufteilung in Unterprogramme als deutlich überdurchschnittlich gekennzeichnet, die sich keineswegs zwingend aus den Vorgaben ergäbe, sondern ein für den Ersteller typisches Stilmerkmal aufweise (Gutachten S. 25 = GA I 159 sowie Vernehmungsprotokoll I 206). Den Feststellungen der Vorinstanzen und der Aussage des Zeugen $\mathrm{H}$. läßt sich nicht entnehmen, daß der Zeuge die in diesem Bereich vorhandenen eigenschöpferischen Züge mitgeprägt hat.

8 = Urt. v. 9. Mai 1985 (I ZR 52/83), Informatik und Recht (iur) 1986, S. 18-23, 22.

9 = Urt. v. 9. Mai 1985 (I ZR 52/83), Informatik und Recht (iur) 1986, S; 18-23, 21. 
Nach alledem kann jedenfalls die Vermutung einer Urheberschaft des Klägers nicht als widerlegt angesehen werden.

c) Allerdings kann der Kläger nach den tatrichterlichen Feststellungen nicht als alleiniger Urheber, sondern gemeinsam mit dem Zeugen $\mathrm{H}$. nur als Miturheber des streitgegenständlichen Computerprogramms angesehen werden. Aus der Aussage des Zeugen H., den das Berufungsgericht für glaubwürdig gehalten hat, ergibt sich, daß er an der Gestaltung des Programms in schöpferischer Weise mitgewirkt hat. Dies gilt zum einen hinsichtlich der Fremdwährungsbuchhaltung. Insoweit hat der Zeuge näher dargelegt, er habe dem Kläger vorgegeben, welche Schritte $\mathrm{zu}$ machen seien, und zwar hinsichtlich der Dateierweiterung, der Plausibilitätsprüfung und der Auswertungsmöglichkeit an bestimmten Punkten. Auch auf die zum schöpferischen Bereich gehörende Datenstrukturierung hat der Zeuge jedenfalls insoweit eingewirkt, als er die Erweiterung der Belegnummerndatei dahingehend vorgeschlagen hat, daß der Schlüssel nicht nur aus der laufenden Belegnummer, sondern auch aus der Mandantennummer und der Kontenart bestand. Außerdem hat der Zeuge nach seinen Bekundungen auch die "Alternative Bilanzgliederung, so konzipiert und entwickelt, $\mathrm{da} ß$ sie in das bestehende Programm integriert werden konnte.

Es kann dahinstehen, ob und ggfs. welche weiteren schöpferischen Beiträge der Zeuge $\mathrm{H}$. geleistet hat. Denn bereits die vorstehend angeführten reichen zur Anerkennung einer Miturheberschaft aus.

4. Ist der Kläger danach nicht alleiniger Urheber, sondern bloßer Miturheber i.S. des $₫ 8$ Abs. 1 UrhG, so folgt daraus, daß er die Ansprüche aus Verletzungen des gemeinsamen Urheberrechts nach $\$ 8$ Abs. 2 Satz 3 UrhG zwar allein geltend machen, grundsätzlich aber nur Leistung an alle Miturheber verlangen kann. Falls es für die weitere Entscheidung darauf ankommt, wird das Berufungsgericht dazu auf die Stellung sachdienlicher Anträge hinzuwirken haben.

5. Die weitere Frage, ob die Beklagte das streitgegenständliche Computerprogramm in einer das Miturheberrecht des Klägers verletzenden Weise verwertet hat, bedarf einer tatrichterlichen Aufklärung. Diese Frage, die Beweisthema des Beschlusses vom 23. Januar 1987 (GA I 75) ist, ist in den Vorinstanzen unbeantwortet geblieben, da es darauf vom Standpunkt der Tatrichter aus nicht mehr ankam. Die Beklagte hat vor allem in ihren Schriftsätzen vom 20. November 1986 und 9. September 1988 (GA I 48 f. und 167 ff.) bestritten, daß das vom Sachverständigen untersuchte Computerprogramm mit dem identisch ist, das sie bei einer Lörracher Firma installiert habe. Allein die Vorlage des Abdrucks der Maske gemäß Anlage K 7, die einerseits die Bezeichnung der Lörracher Firma und andererseits in der Kopfleiste - jedenfalls der ersten beiden Blätter - den Vermerk "REL 2.02/A.S.W،" enthält, reicht nicht zum Nachweis einer urheberrechtsrelevanten Übereinstimmung beider Programme aus, zumal die Blätter 3 und 4 des Maskenausdrucks in der Kopfleiste die Firmenabkürzung der Beklagten "C.P.L." enthalten. In dem wiedereröffneten Berufungsrechtszug werden die Parteien insoweit Gelegenheit zu weiterem Vorbringen haben.
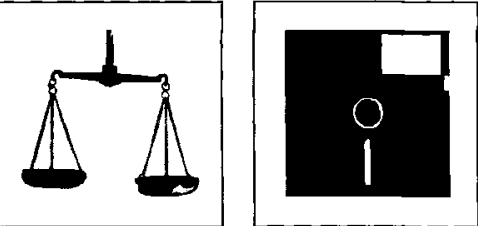

Kläger nicht alleiniger Urbeber, sondern nur Miturheber

Prozessuale Folgen der Miturbeberschaft

Weitere tatrichterliche Aufklärung nötig

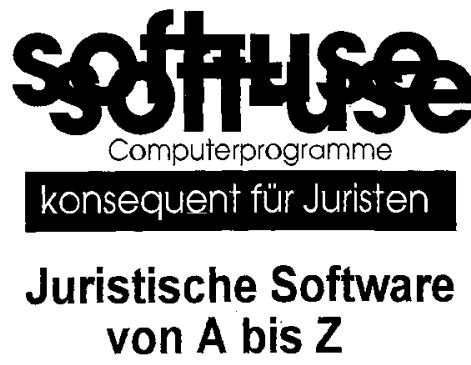

juristisch spezialisierte Settware, CD-ROMs in groBer Auswahl, Hardware und EDV-Zubehör. Beratung. Service. Fordern Sie Infos, Preislisten und den "großen EDV-Katalog für Juristen" an bei:

soft-use Computerprogramme $\mathrm{GmbH}$ Postfach 1153 / 57601 Altenkirchen Tel.: 02681/70468 Fax: 02681/70920 